5-spored asci in both hybrids, as well as a 6-spored ascus in hybrid 66 .

All the spores from a 5-spored ascus of hybrid 65 were isolated, but only four of them germinated. These four gave a segregation ratio of $3 M: 1 \mathrm{~m}$.

A 3M: $1 m$ segregation was also obtained from a 5 -spored ascus of hybrid 66 , in which only four spores germinated.

All ten spores from two 5-spored asci of hybrid 66 germinated, and both asci segregated out as $2 M: 3 \mathrm{~m}$.

Finally, all the spores from a 6-spored ascus of hybrid 66 were isolated, but only four of them germinated and gave a ratio of $3 M: 1 \mathrm{~m}$.

It is thus evident that 8-nucleate asci are produced in this material. When asci of this type produce only four spores, the chances, according to a simple calculation of probabilities, will be that:

\begin{tabular}{|c|c|c|c|c|c|c|}
\hline $\begin{array}{l}51 \cdot 4 \\
22 \cdot 9\end{array}$ & per & & asci & will & segregate & \\
\hline $22 \cdot 9$ & ,", & $"$ & ", & , &, & ," \\
\hline 1.4 & ", & ", & ", & $"$ & ," & ", \\
\hline $1 \cdot 4$ & , & ,", & ", & $"$ & ," & $"$ \\
\hline
\end{tabular}

If, for example, 10 per cent of the 4-spored asci had originally contained eight nuclei, it would thus be expected that $c .4 \cdot 6$ per cent of them would yield either $3: 1$ or $1: 3$ segregations instead of the normal $2: 2$ ratio.

This proof of the occurrence of additional nuclear divisions within the ascus gives, therefore, a simple explanation for the fact that exceptions to classical Mendelian ratios may occur.

Ö. WiNGE

C. ROBERTS

Physiological Department,

Carlsberg Laboratorium, Copenhagen.

'Winge, Ŏ., and Roberts, C., C.R. Lab. Carlsberg, Sér. Physiol., 24, $263(1948)$

"Winkler, H., "Die Konversion der Gene" (1930).

"Lindegren, C. C., "The Yeast Cell, its Genetics and Cytology" (1949).

\section{Carbohydrase Specificity in Saccharomyces Hybrids}

The substrate specificity of the yeast carbohydrases has been studied by many authors ${ }^{1-3}$. It has been concluded $4, \mathbf{s}$ that standard industrial yeastsbrewers', distillers' and bakers' yeasts - contain carbohydrases the substrate specificity of which is determined qualitatively only by the nature of the glucosidic terminal unit of the sugar substrate. On this basis, distinction has been made between the following: (1) a beta-fructofuranosidase which catalyses the removal by hydrolysis of the fructofuranosidic terminal from sucrose, raffinose, stachyose, gentianose and beta-methylfructofuranoside, (2) an alpha-glucosidase which catalyses the removal by hydrolysis of the alpha-glucosidic terminal from maltose, sucrose, turanose, melezitose and suitable synthetic alpha-alkyl glucosides and alpha-aryl glucosides, and (3) an alpha-galactosidase, which catalyses the hydrolyses of the alpha-galactosidic terminal from melibiose, raffinose and alpha-phenyl galactoside.

Enzyme preparations derived by us from haploid hybrid Saccharomyces strains of genetically defined constitution ${ }^{6}$ do not conform with this classification. These yeasts yield an elpha-glucosidase which acts rapidly on alpha-methyl glucoside and is inert both to maltose and some other oligosaccharides with a terminal alpha-glucosidic unit. The haploids have also provided a specific sucrase which acts neither on raffinose nor maltose. It is therefore to be concluded that the number of the yeast carbohydrases is larger and their substrate specificity much narrower than has hitherto been supposed. The differentiation in the yeast material of a specific alpha-methyl glucosidase and a new sucrase provide an apt illustration of the effectiveness of the genetic method.

This research was supported in part by AnheuserBusch, Inc.

Hebrew University, Jerusalem.

$$
\text { SHLOMo HESTRIN }
$$

Carl C. Lindegren

Southern Illinois University, Carbondale, Illinois.

${ }^{1}$ Fischer, E., and Lindner, P., Ber., 28, 3034 (1895).

"Willstätter, R., and Bamann, E., Z. physiol. Chem., 152, 202 (1926).

${ }^{3}$ Euler, H. von, and Brunius, E., Z. physiol. Chem., 160, 242 (1926).

- Leibowitz, J., and Hestrin, S., Biochem. J., 36, 772 (1942).

Weidenhagen, R., and Renner, A., Z. Wirtschaftsgruppe Zuckerind., 86, $22(1936)$.

- Lindegren, Carl C., and Lindegren, Gertrude, Proc. D.S. Nat. Acad. Sci., 35, $23(1949)$.

\section{Occurrence of Unusual Positive Coombs Reactions and $M$ Factors in the Blood of a Mother and Her First Baby}

FollowING routine investigations of blood factors of a woman, aged twenty-four, and her first baby, it was found that the red cells of the mother and her child both gave positive reactions to the Coombs test, when first tested shortly after the birth of the child, and on several occasions during the next eighteen months. The red cells of the father, and those of a second child born fifteen months after the first, gave negative reactions to the Coombs test.

The mother's blood was $A_{1}, R h$-positive $\left(R_{1} R_{1}\right)$, $P$-negative, and when first tested appeared to be type $N$. The first baby was $A_{1}, R h_{\text {-positive }}\left(R_{1} R_{1}\right), P$ negative and type $M$. The father was $A$, $R h_{\text {. }}$. positive $\left(R_{1} R_{1}\right)$ and type $M$. The second baby's blood was $A_{1}, R h$-positive $\left(R_{1} R_{1}\right) M N$, and did not give a positive Coombs test. (The anti-Rh sera. used were anti- $r h^{\prime}$ (anti-C), anti- $R h_{0}$ (anti-D), anti$r h^{\prime \prime}$ (anti-E), and anti- $h r^{\prime}$ (anti-c). It is therefore probable, and has been assumed, that the genotype in each case was $R_{1} R_{1}$ rather than the very rare $R_{1} R^{\prime}$.) The mother's serum did not agglutinate cells containing agglutinogens $R_{1}, R_{2}, r, M, N, P$ or $p$, Lewis-positive or Lewis-negative cells. Kahn reactions were negative in blood of mother and first child.

There was no clinical or laboratory evidence, except the positive Coombs reactions, to suggest hæmolytic disease, either in the mother or the first baby, nor could species reaction to normal rabbit serum be demonstrated. It is therefore suggested that the positive Coombs tests were due to the presence of abnormal receptors of a hereditary nature, and that an unidentified antigen in the pooled human serum used to immunize the rabbits for the production of Coombs reagent had stimulated antibodies reactive with these receptors.

The $M$ - and $N$-types of the mother and first child, when first tested with single samples of potent $M$ and $N$-testing fluids, appeared not to conform with the accepted rule of heredity of these factors, and special tests for the detection of a possible $N_{2}$ factor were negative. Samples of the blood of the mother and 